\title{
Canopy Gaps Improve Landscape Aesthetic Service by Promoting Autumn Colour-Leaved Tree Species Diversity and Colour-Leaved Patch Properties in Subalpine Forests of Southwestern China
}

\section{Qiaoyu Li}

Southwest University

Yu Du

Southwest University

Yuan Liu

Southwest University

Juan Chen

Southwest University

Xiaojing Zhang

Southwest University

Jinchun Liu

Southwest University

Jianping Tao ( $\square$ taojianping@163.com )

sothwest university

\section{Research}

Keywords: biodiversity maintenance, forest aesthetic service functioning, forest stability, gap dynamics, patch structure attributes, species diversity, subalpine autumn colour-leaved forests

Posted Date: December 29th, 2020

DOl: https://doi.org/10.21203/rs.3.rs-135010/v1

License: (c) (1) This work is licensed under a Creative Commons Attribution 4.0 International License. Read Full License 


\section{Abstract}

\section{Background}

Landscape aesthetic service is a crucial ecosystem service in subalpine forests of the mountain and ravine regions of the Tibetan Plateau; it can provide important ecological and economic value, improving the livelihood of the local people. Canopy gap is recognized as the key mechanism in the maintenance of species diversity and forest stability and is known to further affect ecosystem service functioning. However, the mechanisms and interactions of forest stability maintained by gap formations and forest aesthetic service functioning via gap dynamics are not fully understood. We used very high-resolution images to quantify gap characteristics and examined the effects of canopy gaps on autumn colourleaved tree species diversity, colour-leaved patch structure characteristics, and landscape aesthetic service in 21 subalpine autumn colour-leaved forest plots in southwestern China. We then used path analysis to determine the relationships between these factors.

Results

The number of gaps and total gap area were the key gap characteristics affecting landscape aesthetic service; particularly, medium canopy gaps (51-100 $\left.\mathrm{m}^{2}\right)$ played a more important role. The path model showed that increasing the total gap area along with the number of medium canopy gaps had direct positive effects on autumn colour-leaved tree species diversity, colour-leaved patch diversity, and total colour-leaved patch area, thus improving the landscape aesthetic service.

\section{Conclusion}

Canopy gaps indirectly improve landscape aesthetic service by significantly affecting colour-leaved species diversity, colour-leaved patch diversity, and colour-leaved patch area. Our results suggest that forest managers may be able to manipulate the numbers and proportion of medium canopy gaps to protect and preserve autumn colour-leaved tree species diversity and colour-leaved landscapes, promoting the landscape aesthetic service of subalpine autumn colour-leaved forests and, in turn, ensuring the coordinated development of economic, social, and ecological benefits for the underdeveloped rural montane areas of southwestern China.

\section{Background}

Mosaic patches in forests-the result of canopy gap formation and dynamics-play a significant role in the conservation of species diversity, stabilisation of forest landscape structure, and functioning of forest ecosystem services (Watt 1947; Lertzman et al. 1996; Chávez and Macdonald 2010). Canopy gaps are caused by the destruction or mortality of canopy trees or branches by various factors such as old age; pests; and strong snow, wind, or fire (Zang and Wang 2002). Canopy gap formation affects microenvironmental heterogeneity and resource distribution and offers opportunities for tree colonization and regeneration. Over time, partial filling trees gradually reach or approach the canopy layer and form 
relatively closed forest patches. This process is termed gap dynamics or forest-growth-cycle (Brokaw and Busing 2000; Yamamoto 2000). The subalpine forest distributed in the eastern edge of the Tibetan plateau is maintained by this kind of forest-growth-cycle (Wang et al. 2007). A conceptual model has been developed by Wang et al. (2007) to describe the cycle stages in a subalpine forest, which are as follows-the creation of gaps in coniferous forests due to disturbance (which serve as triggers for the forest cycles), the development of broad-leaved patches in the gaps, the regeneration of broad-leaved patches into mixed patches, and finally the return of coniferous forests. Therefore, forest gap dynamics are an important form of forest stability maintenance and species diversity conservation, not only in tropical regions but also in subalpine mountain areas (Zang et al. 2005).

Subalpine autumn colour-leaved forests are widely distributed in the mountain and ravine regions at the eastern edge of the Tibetan Plateau in southwestern China. The stability of forests is often conserved by various mosaic patches (in different gap filling stages), which are formed by gaps with a green background (Wang et al. 2006). These green backgrounds are often dominated by evergreen fir species, and autumn-coloured patches are dominated by deciduous tree species (e.g., birch, maple, poplar, and mountain ash, also called autumn colour-leaved tree species because of their colourful leaves in the autumn season). Previous research in subalpine forests indicated that autumn colour-leaved trees are considered pioneer tree species in the subalpine forest gap succession series, and canopy gaps provide favourable environmental conditions (e.g., high-light environments) for their establishment and regeneration (Wang et al. 2006). Therefore, canopy gaps are an important structure for the existence and maintenance of autumn colour-leaved trees in subalpine autumn colour-leaved forests. Meanwhile, the autumn colour-leaved trees and the colour-leaved patches within canopy gaps display bright colours in autumn that can evoke positive emotional responses in people (Kuper 2018) and play a central role in determining the landscape aesthetic service of subalpine autumn colour-leaved forests. Therefore, canopy gaps play a key role in the supply of landscape aesthetic service in subalpine autumn colourleaved forests; as such, we need a better understanding of how forest stability is maintained by gap formations and how forest aesthetic service functioning is maintained via gap dynamics.

Landscape aesthetic service is a key cultural ecosystem service based on biodiversity (Gobster et al. 2007; Daniel et al. 2012; Tribot et al. 2018), and several studies have shown that landscape aesthetic service correlates positively with species diversity (Lindemann-Matthies and Marty 2013; Tribot et al. 2016). Furthermore, the structural attributes of patches are key factors in determining this ecosystem service (Daniel 2001; Dudek 2018). Many studies have found that colour patch diversity (Zhang et al. 2017), patch shape complexity (Liu et al. 2018), and spatial distribution and proportion of colour patches (Ma et al. 2020) have a strong impact on landscape aesthetic service. The changes in autumn colourleaved tree species diversity and colour-leaved patch structure attributes induced by canopy gaps may have a significant influence on landscape aesthetic service in subalpine autumn colour-leaved forests. Therefore, it is likely that an inherent linkage between forest community stability and forest ecosystem services (landscape aesthetic service) exists by way of canopy refilling processes (gap dynamics). However, very few studies have examined how canopy gaps affect autumn colour-leaved tree species 
diversity and colour-leaved patch structure attributes, thereby affecting landscape aesthetic service in subalpine autumn colour-leaved forests.

An objective and quantifiable evaluation of gap characteristics and patch structure attributes is essential for understanding the effects of canopy gaps on species diversity, patch structure attributes, and related ecosystem services. The traditional method is based on ground measurements at the stand level (Schliemann and Bockheim 2011) and is effective to account for gap area and number, but not to accurately measure other key gap features (e.g. shape) and to adequately reflect the spatial distribution pattern; additionally, surveys over large areas are time-consuming and labour intensive (Nakashizuka et al. 1995). The method based on remote sensing satellite images or aerial photography is thought to be a better way to solve these problems so as to obtain measurements efficiently and accurately at a large scale (Nyamgeroh et al. 2018). However, even the resolution of conventional aerial or satellite images is commonly not high enough to permit accurate spatial quantifications of small canopy gaps or patches (Getzin et al. 2014). With the development of unmanned aerial vehicles with near-surface remote sensing technology, very high-resolution aerial images can be obtained easily, which can then be used to detect small canopy gaps or patches, even those with sizes as small as $1 \mathrm{~m}^{2}$ (Getzin et al. 2012).

In this study, we used very high-resolution images to quantify the characteristics of canopy gaps and colour-leaved patches and examined the effects of gap characteristics on autumn colour-leaved tree species diversity, colour-leaved patch structure attributes, and landscape aesthetic service. Path analysis was used to determine the relationship between canopy gaps and autumn colour-leaved tree species diversity, colour-leaved patch structure attributes, and landscape aesthetic service in 21 subalpine autumn colour-leaved forest plots. The objective of this study was to quantify the effects of canopy gaps on autumn colour-leaved tree species diversity, colour-leaved patch structure attributes, landscape aesthetic service, and their interactions. We hypothesized that autumn colour-leaved tree species diversity, colour diversity, and colour patch area have positive effects on landscape aesthetic service, and canopy gaps positively influence landscape aesthetic service by directly affecting autumn colour-leaved tree species diversity and colour-leaved patch structure properties. We additionally postulated that gap size and gap number were the two most important aspects affecting colour-leaved patch attributes, further affecting landscape aesthetic service.

\section{Materials And Methods}

\section{Study area}

This study was conducted in the subalpine autumn colour-leaved forests of Li County, located in western Sichuan, China $\left(102^{\circ} 41^{\prime}-103^{\circ} 14^{\prime} \mathrm{E}, 31^{\circ} 24^{\prime}-31^{\circ} 50^{\prime} \mathrm{N}\right.$ ) (Figure 1). Elevation ranges from 2000 to $3500 \mathrm{~m}$, with slope ranging from approximately $5-40^{\circ}$. The region is characterized by a monsoon mountain climate, with dry periods from November to April and wet periods from May to October. The annual mean temperature is $8.66^{\circ} \mathrm{C}$, ranging from $0.56{ }^{\circ} \mathrm{C}$ in January to $16.39^{\circ} \mathrm{C}$ in July. The average annual 
precipitation is $772 \mathrm{~mm}$, ranging from 600 to $1100 \mathrm{~mm}, 88 \%$ of which falls during the summer season from May to October (Liu et al. 2018).

The region is famous for the biggest autumn colour-leaved sightseeing forests of China, characterized by high biodiversity and rich tourism resources; ecotourism based on the viewing of autumn colour-leaved forests is one of the major components of the revenue of the area. The vegetation is dominated by evergreen trees (Abies faxoniana, Picea asperata, Quercus aquifolioides) and mixed with autumn colourleaved trees such as Acer oliverianum, Betula platyphylla, Cotinus coggygria, Sorbus koehneana, and Larix kaempferi.

\section{Gap characteristics measurement}

In this study, colour-leaved mosaic patches originated from the filling of canopy gaps in forests, therefore we defined canopy gap in this study as a space filled with autumn colour-leaved trees and surrounded by evergreen trees at the boundary. Canopy gaps were detected and mapped from very high-resolution aerial photographs (approximately $2.5 \mathrm{~cm} /$ pixel). Aerial photos of 21 plots were taken by drones (DJI Mavic air, Shenzhen, China; details of the drones can be found at https://www.dji.com/cn/mavic-air/info\#specs) during autumn leaf-colouration peak periods in October 2018 and 2019 (Zhang et al. 2020). The photos were taken from approximately $50 \mathrm{~m}$ above the centre of the target plots, with the camera perpendicular to the slope, in order to obtain very high-resolution images and to ensure that the shooting area of all plots was approximately $7500 \mathrm{~m}^{2}$. Maps of gaps were manually sketched with visual interpretation to create shape files as accurately as possible using ArcGIS 10.6 (ESRI, Redlands, USA); vector data was subsequently converted to raster data (Figure 2).

Landscape indices have been proven to accurately quantify gap characteristics in previous studies (Getzin et al. 2012; Wu et al. 2016). Three widely used indices were selected to quantify the gap size, shape, and spatial distribution. Gap area represented gap size. Gap shape index estimated gap shape complexity, with the value of the standard shape (square of same size) being 1; the larger the gap shape index value, the more complex the shape. Euclidean nearest-neighbour distance of gaps assessed the degree of aggregation of gaps (the distance to the nearest neighbouring gap); the smaller the value, the more aggregated the gap distribution. These indicators were calculated using FRAGSTATS 4.2.1 (University of Massachusetts, USA), a spatial pattern analysis program used to quantify landscape structure attributes.

\section{Autumn colour-leaved tree species diversity measurement}

Based on the map of gaps at each site, each gap was placed in a $25 \mathrm{~m}^{2}$ quadrat to investigate canopy autumn colour-leaved trees. When the gap area was larger and one quadrat could not represent all autumn colour-leaved tree species within the canopy gap, more quadrats were added to measure as much of the autumn colour-leaved tree species of the canopy gap as possible (details of the number of quadrats in canopy gaps are provided in Table S1). Species name, species number, and leaf colour were recorded in each quadrat from September to October 2019. Considering the inconsistency of quadrat area 
in gaps, the Gleason index (GI) was used to calculate the autumn colour-leaved tree species diversity; the formula for the index is as follows: $\mathrm{GI}=\mathrm{S} / \mathrm{Ln} \mathrm{A}$, where $\mathrm{S}$ is the number of autumn colour-leaved tree species in each gap, and $\mathrm{A}$ is the total survey area of each gap. A total of 35 tree species were noted, and 27 autumn colour-leaved tree species were recorded, including S.koehneana, Rhuspunjabensis, $A$. oliverianum, A. davidii, B. platyphylla, B. utilis, C. coggygria, L. kaempferi, L. mastersiana, etc.

\section{Colour-leaved patch structure attribute measurement}

The colour digital photo method of Zhang et al. (2017) - an objective method to quantify forest colourwas used to measure colour-leaved patch structure attributes. The shooting rules were consistent with canopy gap photography. We digitized each photo and divided it into nineteen colours (black, grey, white, $\mathrm{H} 1, \mathrm{H} 2, \mathrm{H} 3, \mathrm{H} 4, \mathrm{H} 5, \mathrm{H} 6, \mathrm{H} 7, \mathrm{H} 8, \mathrm{H} 9, \mathrm{H} 10, \mathrm{H} 11, \mathrm{H} 12, \mathrm{H} 13, \mathrm{H} 14, \mathrm{H} 15$, and H16) (Figure S1). Analysing the patch colour of all plots, we further found that red, orange, yellow, and green were the main colours of the subalpine autumn colour-leaved forests, so we simplified the colours into eight types: H1 (red), H2 (redorange), H3 (orange), H4 (yellow), H14 (fuchsia), H15 (rose), H16 (magenta), and H0 (green and all other colours) (Figure 3). The processes of photo digitization and colour extraction were performed using MATLAB 2018a (Math Works, Natick, USA) with self-programming (details provided in Zhang et al. (2017)). We converted the map of the colour-leaved patch distribution to a grid using ArcGIS, and the indices of colour-leaved patches were calculated using FRAGSTATS. Four indices were selected to characterise colour-leaved patch structure attributes. Number of colour-leaved patch types represented colour-leaved patch diversity. Colour-leaved patch area represented colour-leaved patch size. Shape index of colour-leaved patch represented colour-leaved patch complexity, and the Euclidean nearest-neighbour distance of colour-leaved patches estimated spatial configuration of colour-leaved patches.

\section{Landscape aesthetic service measurement}

Scenic beauty estimation was used to measure the landscape aesthetic service of subalpine autumn colour-leaved forests (Daniel and Boster 1976). We first selected a photo taken during the autumn leafcolouration peak periods in October 2019 to represent the landscape of a plot; subsequently, 21 photos were selected (one from each plot) to create the electronic questionnaire by Wenjuanxing (a web questionnaire platform). The questionnaire consisted of two parts. The first part of the questionnaire asked the participants for their demographic information, including gender, age, occupation, education, and residence status. The second part asked the participants to evaluate the landscape aesthetic service of subalpine autumn colour-leaved forest by rating each image on a scale ranging from -3 (dislike it very much) to 3 (like it very much). More details about the samples and the questionnaire are given in Appendix S1. The questionnaire was randomly issued with the help of Wenjuanxing through an online survey from October to November 2019 in China. We collected 219 questionnaires, and 179 were ultimately determined to be valid for analysis. Basic demographic information of the participants is provided in Table S2.

\section{Data analysis}


Canopy gaps in each plot were categorised into five gap groups: small gap $\left(\leq 50 \mathrm{~m}^{2}\right)$, medium gap (51$\left.100 \mathrm{~m}^{2}\right)$, large gap $\left(101-500 \mathrm{~m}^{2}\right)$, very large gap (501-1000 $\left.\mathrm{m}^{2}\right)$, and extremely large gap $\left(>1000 \mathrm{~m}^{2}\right)$. We discussed gap characteristics, colour-leaved species diversity, and colour-leaved patch structure attributes at three scales. The first level was that of gaps and included indices such as gap area, gap shape index, and autumn colour-leaved tree species diversity, number of colour-leaved patch types, colour-leaved patch shape index, and colour-leaved patch area of each gap; the second level was that of gap groups and included indices such as total gap area, number of gaps, mean shape index of gaps, mean Euclidean nearest-neighbour distance of gaps, and autumn colour-leaved tree species diversity, number of colour-leaved patch types, mean shape index of colour-leaved patches, total colour-leaved patch area, and the mean Euclidean nearest-neighbour distance of colour-leaved patches of all gaps in each gap group; the third level was that of plots, and consisted of indices including total gap area, number of gaps, mean shape index of gaps, mean Euclidean nearest-neighbour distance of gaps, and autumn colour-leaved tree species diversity, number of colour-leaved patch types, mean shape index of colour-leaved patches, total colour-leaved patch area, and the mean Euclidean nearest-neighbour distance of colour-leaved patches of all gaps in each plot.

The landscape aesthetic service (LAS) measured existing differences in the answers of different participants, affecting the evaluation results according to either strict or loose standards. We used the method reported by Daniel et al. (2001), which was effective in eliminating differences in evaluation results, to calculate the LAS for each plot. The detailed process is as follows:

$$
\begin{gathered}
c p=c f / N, \\
z=\operatorname{NOR}(c p), \\
\bar{z}=\frac{\sum_{n=1}^{n-1} z_{n}}{n},
\end{gathered}
$$

$\operatorname{LAS}=\left(\bar{z}_{i}-\bar{z}_{a}\right) \times 100$

where $c f$ is the cumulative frequency value of each grade ranked from high to low; $c p$ is the cumulative probability value of each grade ranked from high to low; $N$ is the number of questionnaires; NOR is $Z$ is the standard normal deviation (NOR) associated with each of the cumulative probability values; $\bar{z}$ is the arithmetic mean of $z, n$ is grade, $n=1, \ldots, 7$ corresponding to the score from -3 to $3 ; \bar{z}_{i \text { is }}$ the arithmetic average of $z$ with the ith landscape; and $\bar{z}_{a}$ is the arithmetic average of $z$ with a landscape by random selection.

Normality of all variables was checked using the Kolmogorov-Smirnov test. We tested for variations in gap characteristics, autumn colour-leaved tree species diversity, and colour-leaved patch structure attributes among five gap size classes and among five gap groups using Kruskal-Wallis one-way analysis of variance because of heterogeneous variances and unequal sample numbers between five gap size 
classes and five gap groups. Meanwhile, correlation analyses were used to determine the relationships between canopy gaps with autumn colour-leaved tree species diversity and colour-leaved patch structure attributes at the gap level and at the gap group level. Gap area, colour-leaved patch diversity, and colourleaved patch area were natural log-transformed for all analyses.

To test the effects of canopy gaps on landscape aesthetic service, colour-leaved species diversity and colour-leaved patch structure attributes, and their interactions, were examined. First, we used correlation analysis to determine the relationships between landscape aesthetic service and gap characteristics, colour-leaved species diversity, and colour-leaved patch structure attributes. Then, path analysis was used to test how canopy gaps affected landscape aesthetic service by affecting autumn colour-leaf tree species diversity and colour-leaved patch structure attributes. Models were assessed by Chi-square goodness-of-fit ( $p>0.05$ indicate good fit), standardized root mean square residual (SRMR; values $<0.08$ indicate good fit), and comparative fit index (CFl; values $>0.95$ indicate good fit) (Grace et al. 2010). We aimed to determine how canopy gaps affected landscape aesthetic service and which size of canopy gaps played a more important role in landscape aesthetic service in subalpine autumn colour-leaved forests. Thus, the effects of canopy gaps on landscape aesthetic service were examined at gap group levels and at plot levels.

Statistical analyses were performed using SPSS 24.0 (IBM, Armonk, USA), and path analyses were performed using AMOS 23.0 software (IBM, Armonk, USA).

\section{Results}

\section{Gap characteristics}

The percentage of total forest area covered by gaps was $21.69 \%$. A total of 403 gaps were detected, with a total gap area of $35108 \mathrm{~m}^{2}$. The average gap area was $87.18 \pm 13.63 \mathrm{~m}^{2}$ (mean $\pm \mathrm{SE}$ ), ranging from 1 to $3468 \mathrm{~m}^{2}$ (Table S3). The gap size distribution decreased exponentially, in terms of number of gaps, with increasing size (Table S3). Of the total number of gaps, more than $70 \%$ were $\leq 50 \mathrm{~m}^{2}$ in size. The gap number distribution of five different size classes showed that more than half of the plots were dominated by gaps $\leq 50 \mathrm{~m}^{2}$ in size (Table S3). The proportions of gap area in the five size classes showed that more than half of the gap area was contributed by gaps $>50 \mathrm{~m}^{2}$ in size, except for several plots that were absolutely dominated by gaps $\leq 50 \mathrm{~m}^{2}$ in size (Figure S2). The average of the mean shape index of gaps (GMSI) was $1.74 \pm 0.04$, with maximum and minimum values of 1.39 and 2.02, respectively (Table S3). The average of the mean Euclidean nearest-neighbour distance of gaps (GMENN) was $4.59 \pm 0.73 \mathrm{~m}$, ranging from 0.00 to $13.98 \mathrm{~m}$ (Table $\mathrm{S} 3$ ).

\section{Effects of canopy gaps on autumn colour-leaved tree species diversity and colour-leaved patch structure attributes}


At the gap level, gap shape index, autumn colour-leaved tree species diversity (ACTSD), and colour-leaved patch structure attributes of the five canopy gap-size classes exhibited a large variation (all $p<0.001$ ), and these indicators were significantly higher in gaps $>50 \mathrm{~m}^{2}$ in size than in gaps $\leq 50 \mathrm{~m}^{2}$ in size (Table 1). Correlation analysis results showed that the ACTSD (Figure 4a; $r=0.568, p<0.01$ ), colour-leaved patch diversity (CPD) (Figure 4b; $r=0.608, p<0.01$ ), colour-leaved patch area (CA) (Figure 4d; $r=0.962, p$ $<0.01$ ), gap shape index (GSI) (Figure 4e; $r=643, p<0.01$ ), and colour-leaved patch shape index (CSI) (Figure 4f; $r=542, p<0.01$ ) significantly increased with increasing gap area (GA). CPD was positively related to ACTSD (Figure 4c; $r=0.380, p<0.05$ ), and CSI significantly increased with increasing GSI (Figure 4g; $r=0.552, p<0.01$ ).

Table 1 Differences of gap characteristics, colour-leaved tree species diversity, colour-leaved patch structure attributes in five gap size classes.

\begin{tabular}{|lllllll|}
\hline & $\leq 50 \mathrm{~m}^{2}$ & $51-100 \mathrm{~m}^{2}$ & $101-500 \mathrm{~m}^{2}$ & $501-1000 \mathrm{~m}^{2}$ & $>1000 \mathrm{~m}^{2}$ & $p$ \\
\hline GSI & $1.57 \pm 0.01 \mathrm{c}$ & $1.83 \pm 0.05 \mathrm{~b}$ & $1.91 \pm 0.06 \mathrm{ab}$ & $1.94 \pm 0.17 \mathrm{ab}$ & $2.78 \pm 0.30 \mathrm{a}$ & $\mathbf{0 . 0 0 0}$ \\
ACTSD & $0.35 \pm 0.01 \mathrm{c}$ & $0.51 \pm 0.03 \mathrm{~b}$ & $0.60 \pm 0.04 \mathrm{ab}$ & $0.74 \pm 0.14 \mathrm{ab}$ & $1.00 \pm 0.12 \mathrm{a}$ & $\mathbf{0 . 0 0 0}$ \\
CPD & $0.84 \pm 0.03 \mathrm{~b}$ & $1.25 \pm 0.04 \mathrm{a}$ & $1.29 \pm 0.05 \mathrm{a}$ & $1.35 \pm 0.12 \mathrm{a}$ & $1.60 \pm 0.06 \mathrm{a}$ & $\mathbf{0 . 0 0 0}$ \\
CA & $2.14 \pm 0.06 \mathrm{c}$ & $4.25 \pm 0.03 \mathrm{~b}$ & $5.37 \pm 0.07 \mathrm{ab}$ & $6.59 \pm 0.09 \mathrm{ab}$ & $7.42 \pm 0.14 \mathrm{a}$ & $\mathbf{0 . 0 0 0}$ \\
CSI & $1.57 \pm 0.02 \mathrm{~b}$ & $1.88 \pm 0.05 \mathrm{a}$ & $1.84 \pm 0.07 \mathrm{a}$ & $1.83 \pm 0.07 \mathrm{a}$ & $1.78 \pm 0.04 \mathrm{a}$ & $\mathbf{0 . 0 0 0}$ \\
\hline
\end{tabular}

Values are the mean \pm SE (standard error). ACTSD: Autumn colour-leaved tree species diversity; CPD: Colour-leaved patch diversity; CSI: Colour-leaved patch shape index; GSI: Gap shape index; CA: Colourleaved patch area. The $p$-values indicate the results of the Kruskal-Wallis one-way analysis of variance comparing the different gap size classes. Significant results $(p<0.05)$ are highlighted in bold. Values with different letters in the same row mean significant differences among different gap size classes. Same below.

In the five gap groups with different sizes, the number of gaps (GN), total gap area (TGA), GMSI, total colour-leaved patch area (TCA), the mean shape index of colour-leaved patches (CMSI), and the mean Euclidean nearest-neighbour distance of colour-leaved patches (CENN) (all $p<0.01)$ were significantly different (Table 2). There was no significant difference in GENN, ACTSD, and CPD among the five gap groups (Table 2). Correlation analysis results showed that TGA and TCA increased significantly with GN in small (Figure 5a; $r=0.728, p<0.01$; Figure $5 \mathrm{~d} ; r=0.731, p<0.01$ ), medium (Figure $5 \mathrm{a} ; r=0.919, p<$ 0.01; Figure 5d; $r=0.876, p<0.01$ ), and large (Figure 5a; $r=0.769, p<0.01$; Figure $5 \mathrm{~d} ; r=0.687, p<0.01$ ) gap groups. ACTSD and CPD increased significantly with increasing GN in small (Figure $5 \mathrm{~b} ; r=0.473, p<$ 0.05 ; Figure $5 c ; r=0.703, p<0.01$ ) and medium (Figure $5 \mathrm{~b} ; r=0.654, p<0.01$; Figure $5 \mathrm{c} ; r=0.635, p<$ $0.05)$ gap groups. ACTSD of the medium and large groups was positively correlated with TGA (Figure $5 \mathrm{e}$; $r=0.528, p<0.05 ; r=0.551, p<0.05$, respectively). CPD was significantly increased with TGA in small (Figure $5 f ; r=0.669, p<0.01$ ) and medium (Figure $5 f ; r=0.576, p<0.05$ ) gap groups. There was a 
significant positive correlation between TGA and TCA of small, medium, and large gap groups (Figure $5 \mathrm{~g}$; $r=0.924 ; r=923 ; r=0.920$; all $p<0.01$ ). We found a significant relationship between ACTSD and CPD in the small gap group only (Figure $5 \mathrm{j} ; r=0.532, p<0.05$ ). There was no significant relationship between GMSI and CMSI, GMENN and CMENN in the five gap groups.

Table 2 Differences of gap characteristics, colour-leaved tree species diversity and colour-leaved patch structure attributes in five gap groups.

\begin{tabular}{|lllllll|}
\hline & $S$ & $M$ & $L$ & $V L$ & $E L$ & $p$ \\
\hline GN & $14.70 \pm 3.57 \mathrm{a}$ & $3.50 \pm 0.53 \mathrm{~b}$ & $2.60 \pm 0.42 \mathrm{bc}$ & $1.40 \pm 0.24 \mathrm{bc}$ & $1.00 \pm 0.00 \mathrm{c}$ & $\mathbf{0 . 0 0 0}$ \\
\hline TGA & $4.88 \pm 0.25 \mathrm{c}$ & $5.32 \pm 0.18 \mathrm{bc}$ & $6.18 \pm 0.20 \mathrm{ab}$ & $6.91 \pm 0.14 \mathrm{a}$ & $7.42 \pm 0.14 \mathrm{a}$ & $\mathbf{0 . 0 0 0}$ \\
\hline GMSI & $1.64 \pm 0.03 \mathrm{~b}$ & $1.89 \pm 0.06 \mathrm{a}$ & $1.92 \pm 0.09 \mathrm{a}$ & $2.04 \pm 0.21 \mathrm{a}$ & $2.77 \pm 0.36 \mathrm{a}$ & $\mathbf{0 . 0 0 0}$ \\
\hline GMENN & $13.92 \pm 3.4$ & $18.15 \pm 4.12$ & $25.31 \pm 4.74$ & $24.99 \pm 20.42$ & - & 0.131 \\
\hline ACTSD & $0.52 \pm 0.05$ & $0.50 \pm 0.07$ & $0.51 \pm 0.05$ & $0.46 \pm 0.12$ & $0.64 \pm 0.08$ & 0.626 \\
\hline CPD & $1.34 \pm 0.08$ & $1.43 \pm 0.08$ & $1.43 \pm 0.08$ & $1.29 \pm 0.16$ & $1.60 \pm 0.06$ & 0.306 \\
\hline TCA & $4.40 \pm 0.28 \mathrm{c}$ & $4.88 \pm 0.19 \mathrm{c}$ & $5.64 \pm 0.21 \mathrm{~b}$ & $6.42 \pm 0.10 \mathrm{ab}$ & $6.93 \pm 0.13 \mathrm{a}$ & $\mathbf{0 . 0 0 0}$ \\
\hline CMSI & $1.59 \pm 0.03 \mathrm{~b}$ & $1.80 \pm 0.11 \mathrm{a}$ & $1.78 \pm 0.05 \mathrm{a}$ & $1.72 \pm 0.07 \mathrm{ab}$ & $1.76 \pm 0.03 \mathrm{a}$ & $\mathbf{0 . 0 0 6}$ \\
\hline CMENN & $3.98 \pm 1.62 \mathrm{a}$ & $2.01 \pm 0.69 \mathrm{ab}$ & $0.55 \pm 0.08 \mathrm{~b}$ & $0.40 \pm 0.09 \mathrm{~b}$ & $0.59 \pm 0.13 \mathrm{~b}$ & $\mathbf{0 . 0 0 1}$ \\
\hline
\end{tabular}

Values are the mean \pm SE (standard error). The $p$-values indicate the results of the Kruskal-Wallis oneway analysis of variance comparing the different gap groups. $S$ is small gap group; $M$ is medium gap group; $L$ is large gap group; $V L$ is very large gap group; $E L$ is extreme large gap group. ACTSD: Autumn colour-leaved tree species diversity; CMENN: Mean Euclidean nearest-neighbour distance of colour-leaved patches; CMSI: Mean shape index of colour-leaved patch; CPD: Colour-leaved patch diversity; GMENN: Mean Euclidean nearest-neighbour distance of gaps; GMSI: Mean shape index of gaps; GN: Number of gaps; TCA: Total colour-leaved patch area; TGA: Total gap area. '-' means no data.

\section{Effects of canopy gaps on landscape aesthetic service}

Landscape aesthetic service (LAS) was related to all gap groups but was significantly positively related to GN (Figure 6a; $r=0.827, p<0.01$ ), TGA (Figure 6b; $r=0.798 ; p<0.01$ ), CPD (Figure 6f; $r=0.501, p<0.05$ ), and TCA (Figure $6 \mathrm{~g} ; r=0.715, p<0.01$ ) in only the medium-gap group. There was no significant correlation between LAS and ACTSD, GMSI, GMENN, CMSI, and CMENN in all gap groups.

The path model that included the GN, TGA, ACSTD, CPD and TCA using medium-gap group data was a good fit $\left(X^{2}=13.59 ; p=0.09 ;\right.$ SRMR $\left.=0.00 ; C F I=1.00\right)$ and explained $75 \%$ of the variance in LAS (Figure 8a). The model showed that GN had a strong positive correlation with TGA $(\lambda=0.98 ; p<0.001)$, and the increase in TGA indirectly affected LAS by significantly increasing TCA $(\lambda=0.99 ; p<0.001)$, followed by 
$\operatorname{ACTSD}(\lambda=0.66 ; p<0.05)$ and CPD $(\lambda=0.61 ; p>0.05)$. The TCA had a significantly positive direct effect on LAS $(\lambda=0.79 ; p<0.01)$, while the CPD was positively (but not significantly) related to LAS. ACTSD had an indirect effect on LAS by having a direct effect on CPD.

At the plot level, the LAS had a significant positive linkage with GN (Figure 7a; $r=0.461, p<0.05$ ), TCA (Figure 7b; $r=0.609, p<0.01$ ), ACTSD (Figure 7e; $r=0.542, p<0.05$ ), CPD (Figure 7f; $r=0.604, p<0.01$ ), and TCA (Figure 7g; $r=0.580, p<0.01$ ), but there was no significant correlation between LAS and MSI of gaps, GMENN, CMSI, and CMENN.

The path model based on GN, TGA, ACTSD, CPD and TCA at the plot level was a good fit $\left(\chi^{2}=3.74 ; p=\right.$ $0.44 ;$ SRMR $=0.00 ; \mathrm{CFI}=1.00$ ) and explained $61 \%$ of the variance in LAS (Figure $8 b$ ). The model showed that TCA $(\lambda=0.43 ; p<0.05)$ directly and positively affected LAS. The effects of ACTSD $(\lambda=0.10 ; p>$ $0.05)$ and CPD $(\lambda=0.42 ; p>0.05)$ were positive but weak. The TGA had a direct, significant, and positive effect on TCA $(\lambda=0.98 ; p<0.001)$, followed by ACTSD $(\lambda=0.42 ; p<0.05)$. GN had a direct, strong, positive effect on $\operatorname{CPD}(\lambda=0.61 ; p<0.001)$.

\section{Discussion}

\section{Impact of canopy gaps on autumn colour-leaved tree species diversity and colour-leaved patch structure attributes}

Gap size distribution in subalpine autumn colour-leaved forests followed a negative exponential model, with many small canopy gaps and few larger ones (Table S3). This result, obtained by using the highresolution aerial photography method, is similar to the results of a ground survey, where small canopy gaps were shown to be dominant in subalpine forests (Liu and Wu 2002; Wang et al. 2004). Based on the gap-size distribution found in our study, we conclude that the subalpine autumn colour-leaved forests in western Sichuan are shaped by a small-scale disturbance regime, and canopy gaps formed by single or few canopy tree deaths are dominant.

Gap size had a significant impact on species diversity (Menges et al. 2008). In the subalpine autumn colour-leaved forests examined in this study, autumn colour-leaved tree species diversity increased with increasing gap area at the gap level (Figure 4). Similar results have been reported in other forests, where species diversity has been shown to be positively correlated with gap area (Mallik et al. 2014; Weber et al. 2014). In forests, environmental heterogeneity and resource availability often have a strong and positive impact on species composition and diversity (Busing and White 1997; Xu et al. 2016; Kumar et al. 2018). Gap size is a suitable index to characterise microenvironmental heterogeneity and resource sequestration within canopy gaps (Denslow 1987). Large canopy gaps provide more heterogeneous microenvironments and resource availability and support more varied species assemblages (Hubbell et al. 1999; Dee and Menges 2014). In addition, tree species with different levels of shade tolerance respond differently to gap size (Whitmore 1989; Hubbell et al. 1999). Previous studies have suggested that large canopy gaps have higher light availability and contain a higher number of shade-intolerant (early successional) species 
compared to small canopy gaps (Brokaw and Scheiner 1989; Schnitzer and Carson 2001). Thus, it is not surprising that a large canopy gap has a higher number of autumn colour-leaved tree species.

Although species diversity had a significant response to variation in gap area at the gap level, there was no significant difference in autumn colour-leaved tree species diversity among the five gap groups, despite the large variation in total gap area (Table 2). In this study, a majority of plots were characterized by a large number of small canopy gaps and a small number of large canopy gaps, and a large area proportion of large gaps and a small area proportion of small ones. There is no doubt that large-gap groups have a high number of species due to the species-area relationship (Preston 1962); for small gap groups, gap area limits the number of species. The number of gaps plays a key role in determining the opportunities for colonization of trees. We speculate that a large number of small canopy gaps may increase the chances of colonization of different species and thus offset the area limitation of species diversity. Our findings support the hypothesis that autumn colour-leaved tree species diversity was significantly increased with increasing number of gaps in small ( $\leq 50 \mathrm{~m}^{2}$ in size) and medium (51-100 $\mathrm{m}^{2}$ in size) gap groups (Figure 5). This may explain the lack of significant differences in autumn colourleaved tree species diversity among the five gap groups. Thus, when exploring the effects of canopy gaps on species diversity at large scales, the number of gaps should also be considered in addition to gap size.

Species composition and structure within canopy gaps play a key role in determining patch structure attributes (Busing 1998; Manabe et al. 2009). Plant colour diversity often reflects the species diversity experienced visually by humans (Grose 2012). In this study, there was a positive relationship between colour-leaved patch diversity and autumn colour-leaved tree species diversity, and both increased with gap area. This means that canopy gaps not only maintain species diversity but also colour diversity, especially in large canopy gaps. The patch area largely reflects the abundance and total basal area of trees within canopy gaps. Previous studies demonstrated that increasing gap area increased both the number of stems and total basal area (Runkle 1982; Arriaga 2000). Our results showed that colour-leaved patch area exerted a strong positive relationship with gap area. This indicates that large canopy gaps support more autumn colour-leaved tree survival and help maintain the patches formed in them.

\section{Influences of canopy gaps on landscape aesthetic service are mediated through colour-leaved species diversity, colour-leaved patch diversity, and colour-leaved patch area}

Path models demonstrated that the total colour-leaved patch area induced by canopy gaps had a significant influence on landscape aesthetic service. The proportion of colour is known to have a significant impact on landscape aesthetic service (Ma et al. 2020). A large total colour-leaved patch area can form a high visual impact and strong visual traction in forests with green matrices, thereby significantly improving landscape aesthetic service (Li 2018). A high area proportion of canopy gaps in subalpine autumn colour-leaved forests can provide more space for colonization and regeneration of autumn colour-leaved trees. More autumn colour-leaved trees reach the canopy and form a large area of colour-leaved landscapes, thus promoting the landscape aesthetic service. Compared to medium and large canopy gaps, the colour-leaved patches produced in small canopy gaps do not create impressive 
visual impacts, and with low visual attractiveness, they cannot cause strong and positive emotional reactions from appreciators. In addition, canopy gaps caused by small-scale disturbances are dominant, and large or very large canopy gaps are few, which may be the reason why we did not find significant effects of large or very-large gap groups. Thus, based on our results, we suggest that medium canopy gaps play a more significant role in landscape aesthetic service in subalpine autumn colour-leaved forests.

Canopy gaps can also improve the landscape aesthetic service indirectly by increasing the autumn colour-leaved tree species diversity and colour-leaved patch diversity. Previous studies have suggested that species diversity has a positive relationship with landscape aesthetic service (Gobster et al. 2007; Quijas et al. 2012). Our results are consistent with the findings in grasslands (Lindemann-Matthies et al. 2010) and marine ecosystems (Tribot et al. 2016), where landscape aesthetic service was observed to increase with increasing species diversity. These findings provide evidence that landscape aesthetic service has a consistent positive response to species diversity in different ecosystems. Our findings are consistent with the findings in meadows (Hoyle et al. 2018), where the role of colour diversity in landscape aesthetic service was stronger than that of species diversity. This is mainly because species diversity per se is not a driver of human response (Graves et al. 2017), but it directly affects colour diversity and thus establishes a positive linkage with landscape aesthetic service (Lindemann-Matthies et al. 2010). Path models showed that autumn colour-leaved tree species diversity significantly increased with increasing gap area and positively affected colour-leaved patch diversity. Overall, these results highlight the importance of canopy gaps in maintaining species diversity, which allows for colour diversity to improve landscape aesthetic service in subalpine autumn colour-leaved forests.

\section{Conclusions}

Our study is one of the first to demonstrate the impact of canopy gaps on autumn colour-leaved tree species diversity, colour-leaved patch structure attributes, landscape aesthetic service, and their interactions, in subalpine autumn colour-leaved forests. This study offers new insight into the role of canopy gaps in providing landscape aesthetic service. The findings confirm that canopy gaps play a key role in landscape aesthetic service in subalpine autumn colour-leaved forests. Based on our results, we suggest that canopy gaps formed by small-scale disturbances should be reserved or created in subalpine autumn colour-leaved forests that have high canopy closures and/or are dominated by evergreen trees; this allows autumn colour-leaved tree species to regenerate (or be planted), which is beneficial for preserving the trees and the colourful patches they form. Although a large canopy gap is more conducive to the survival of autumn colour-leaved tree species and the production of spectacular colour-leaved landscapes, large-scale disturbances are rare in forests. A large number of small and medium canopy gaps also have high species diversity, and many medium canopy gaps result in a large total gap area and form a striking colour-leaved landscape, thus significantly improving landscape aesthetic service. It is worth noting that small canopy gaps are generally unfriendly to shade-intolerant species as light requirements are not sufficiently met (Cowell et al. 2010; Petritan et al. 2013). Therefore, intermediate 
canopy gaps (51-100 $\mathrm{m}^{2}$ ) are more suitable for the management application of subalpine autumn colour-leaved forests. Increasing the amount of medium canopy gaps is conducive to increasing colourleaved species diversity and maintaining colour-leaved landscape structure, helping to maintain subalpine autumn colour-leaved forests at the optimal stage of higher species diversity and higher landscape aesthetic service, which is of great significance for maintaining ecological security and economic development of the region. The results of this study can help forest managers to conserve tree species diversity, maintain landscape structure, and improve landscape aesthetic service through canopy gap management in subalpine autumn colour-leaved forests.

\section{Abbreviations}

ACTSD: Autumn colour-leaved tree species diversity; CA: Colour-leaved patch area; CENN: Euclidean nearest-neighbour distance of colour-leaved patches; CMENN: Mean Euclidean nearest-neighbour distance of colour-leaved patches; CMSI: Mean shape index of colour-leaved patch; CPD: Colour-leaved patch diversity; CSI: Colour-leaved patch shape index; GA: Gap area; GENN: Euclidean nearest-neighbour distance of gaps; GMENN: Mean Euclidean nearest-neighbour distance of gaps; GMSI: Mean shape index of gaps; GN: Number of gaps; GSI: Gap shape index; LAS: Landscape aesthetic service; TCA: Total colourleaved patch area; TGA: Total gap area.

\section{Declarations}

\section{Acknowledgements}

We would like to thank Sadichhya for her assistance with English language and grammatical editing.

\section{Authors' contributions}

Qiaoyu Li and Jianping Tao conceived the ideas and designed methodology; Qiaoyu Li, Juan Chen and Xiaojing Zhang collected the data; Qiaoyu Li, Yu Du, Yuan Liu, Jinchun Liu and Jianping Tao analyzed the data, Qiaoyu Li, Yuan Liu and Jianping Tao co-wrote the manuscript. All authors contributed critically to the drafts and approved the final version of the manuscript.

\section{Funding}

This manuscript was funded by the National Key Research and Development Program of China (2017YFC0505003).

\section{Availability of data and materials}

The datasets analyzed during the current study are available from the corresponding author on reasonable request. 
Not applicable.

\section{Consent for publication}

Not applicable.

\section{Competing interests}

The authors declare that they have no competing interests.

\section{References}

Arriaga L (2000) Gap-building-phase regeneration in a tropical montane cloud forest of north-eastern Mexico. J Trop Ecol, 16, 535-562. doi: 10.1017/S0266467400001565

Brokaw N V L, Scheiner S M (1989) Species composition in gaps and structure of a tropical forest. Ecology, 70, 538-541. doi: 10.2307/1940196

Brokaw N, Busing R T (2000) Niche versus chance and tree diversity in forest gaps. Trends Ecol Evol, 15, 183-188. doi: 10.1016/S0169-5347(00)01822-X

Busing R T (1998) Composition, Structure and Diversity of Cove Forest Stands in the Great Smoky Mountains: A Patch Dynamics Perspective. J Veg Sci, 9, 881-890. doi: 10.2307/3237053

Busing R T, White P S (1997) Species diversity and small-scale disturbance in an old-growth temperate forest: a consideration of gap partitioning concepts. Oikos, 78, 562-568. doi: 10.2307/3545618

Chávez V, Macdonald S E (2010) The influence of canopy patch mosaics on understory plant community composition in boreal mixedwood forest. Forest Ecol Manag, 259, 1067-1075. doi:

org/10.1016/j.foreco.2009.12.013

Cowell C M, Hoalst-Pullen N, Jackson M T (2010) The limited role of canopy gaps in the successional dynamics of a mature mixed Quercus forest remnant. J Veg Sci, 21, 201-212. doi: 10.1111/j.16541103.2009.01137.x

Daniel T C (2001) Whither scenic beauty? Visual landscape quality assessment in the 21st century. Landscape Urban Plan, 54, 267-281. doi: org/10.1016/S0169-2046(01)00141-4

Daniel T C, Boster R S (1976) Measuring landscape esthetics: the scenic beauty estimation method. USDA Forest Service Research Paper, USA.

Daniel T C, Muhar A, Arnberger A, Aznar O, Boyd J W, Chan K M A, Costanza R, Elmqvist T, Flint C G, Gobster P H, Gret-Regamey A, Lave R, Muhar S, Penker M, Ribe R G, Schauppenlehner T, Sikor T, Soloviy I, Spierenburg M, Taczanowska K, Tam J, Von der Dunk A (2012) Contributions of cultural services to the 
ecosystem services agenda. Proceedings of the National Academy of Sciences, 109, 8812-8819. doi: 10.1073/pnas. 1114773109

Dee J R, Menges E S (2014) Gap ecology in the Florida scrubby flatwoods: effects of time-since-fire, gap area, gap aggregation and microhabitat on gap species diversity. J Veg Sci, 25, 1235-1246. doi: $10.1111 /$ jvs. 12170

Denslow J (1987) Tropical rainforest gaps and tree species diversity. Annual Review of Ecology and Systematics, 18, 431-451. doi: 10.1146/annurev.ecolsys.18.1.431

Dudek T (2018) Influence of selected features of forests on forest landscape aesthetic value- example of Se Poland. J Environ Eng Landsc, 26, 275-284. doi: 10.3846/jeelm.2018.6268

Getzin S, Nuske R, Wiegand K (2014) Using unmanned aerial vehicles (UAV) to quantify spatial gap patterns in forests. Remote Sens-Basel, 6, 6988-7004. doi: 10.3390/rs6086988

Getzin S, Wiegand K, Schöning I (2012) Assessing biodiversity in forests using very high-resolution images and unmanned aerial vehicles. Methods Ecol Evol, 3, 397-404. doi: 10.1111/j.2041210X.2011.00158.x

Gobster P H, Nassauer J I, Daniel T C, Fry G (2007) The shared landscape: what does aesthetics have to do with ecology? Landscape Ecol, 22, 959-972. doi: 10.1007/s10980-007-9110-x

Grace J, Anderson T, Olff H, Scheiner S (2010) On the specification of structural equation models for ecological systems. Ecol Monogr, 80, 67-87. doi: 10.2307/27806874

Graves R A, Pearson S M, Turner M G (2017) Species richness alone does not predict cultural ecosystem service value. PNAS, 114, 3774-3779. doi: 10.1073/pnas.1701370114

Grose M J (2012) Plant colour as a visual aspect of biological conservation. Biol Conserv, 153, 159-163. doi: 10.1016/j.biocon.2012.05.008

Hoyle H, Norton B, Dunnett N, Richards J P, Russell J M, Warren P (2018) Plant species or flower colour diversity? Identifying the drivers of public and invertebrate response to designed annual meadows. Landscape Urban Plan, 180, 103-113. doi: 10.1016/j.landurbplan.2018.08.017

Hubbell S P, Foster R B, O'Brien S T, Harms K E, Condit R, Wechsler B, Wright S J, S. L D L (1999) Light-gap disturbances, recruitment limitation, and tree diversity in a neotropical forest. Science, 283, 554-557. doi: 10.1126/science.283.5401.554

Kumar P, Chen H Y H, Thomas S C, Shahi C (2018) Linking resource availability and heterogeneity to understorey species diversity through succession in boreal forest of Canada. J Ecol, 106, 1266-1276. doi: $10.1111 / 1365-2745.12861$ 
Kuper R (2018) Effects of flowering, foliation, and autumn colors on preference and restorative potential for designed digital landscape models. Environ Behav, 52, 1-13. doi: 10.1177/0013916518811424

Lertzman K P, Sutherland G D, Inselberg A, Saunders S C (1996) Canopy gaps and the landscape mosaic in a coastal temperate rain forest. Ecology, $77,1254-1270$. doi: $10.2307 / 2265594$

Li P (2018) The influence of space characteristics of color patch on the autumn aesthetic quality of the forest of Cotinus coggygria. Matster Matster Thesis, Beijing Forestry University.

Lindemann-Matthies P, Junge X, Matthies D (2010) The influence of plant diversity on people's perception and aesthetic appreciation of grassland vegetation. Biol Conserv, 143, 195-202. doi:

10.1016/j.biocon.2009.10.003

Lindemann-Matthies P, Marty T (2013) Does ecological gardening increase species richness and aesthetic quality of a garden? Biol Conserv, 159, 37-44. doi: 10.1016/j.biocon.2012.12.011

Liu J, Chamberlain B C, Kozak R A, Meitner M J, Nesbitt L (2018) Relating shape to human aesthetic evaluations of harvest blocks from an aerial perspective. Scand J Forest Res, 33, 793-799. doi:

$10.1080 / 02827581.2018 .1527943$

Liu Q, Wu Y (2002) Effects of gap size on regeneration of subalpine coniferous forests in northwest Yunnan. Chinese Journal of Applied and Environmental Biology, 8, 453-459. doi: 10.3321/j.issn:1006687X.2002.05.002

Liu Y, Zhang J, Yang W, Wu F, Xu Z, Tan B, Zhang L, He X, Guo L (2018) Canopy gaps accelerate soil organic carbon retention by soil microbial biomass in the organic horizon in a subalpine fir forest. Appl Soil Ecol, 125, 169-176. doi: 10.1016/j.apsoil.2018.01.002

Ma B, Hauer R J, Xu C (2020) Effects of design oroportion and distribution of color in urban and suburban green space planning to visual aesthetics quality. Forests, 11,278. doi: 10.3390/f11030278

Mallik A U, Kreutzweiser D P, Spalvieri C M (2014) Forest regeneration in gaps seven years after partial harvesting in riparian buffers of boreal mixedwood streams. Forest Ecol Manag, 312, 117-128. doi: 10.1016/j.foreco.2013.10.015

Manabe T, Shimatani K, Kawarasaki S, Aikawa S, Yamamoto S (2009) The patch mosaic of an old-growth warm-temperate forest: patch-level descriptions of 40-year gap-forming processes and community structures. Ecol Res, 24, 575-586. doi: 10.1007/s11284-008-0528-7

Menges E S, Craddock A, Salo J, Zinthefer R, Weekley C W (2008) Gap ecology in Florida scrub: Species occurrence, diversity and gap properties. J Veg Sci, 19, 503-514. doi: 10.3170/2008-8-18399

Nakashizuka T, Katsuki T, Tanaka H (1995) Forest canopy structure analyzed by using aerial photographs. Ecol Res, 1, 13-18. doi: 10.1007/BF02347651 
Nyamgeroh B B, Groen T A, Weir M J C, Dimov P, Zlatanov T (2018) Detection of forest canopy gaps from very high resolution aerial images. Ecol Indic, 95, 629-636. doi: 10.1016/j.ecolind.2018.08.011

Petritan A M, Nuske R S, Petritan I C, Tudose N C (2013) Gap disturbance patterns in an old-growth sessile oak (Quercuspetraea L.)-European beech (Fagussylvatica L.) forest remnant in the Carpathian Mountains, Romania. Forest Ecol Manag, 308, 67-75. doi: 10.1016/j.foreco.2013.07.045

Preston F W (1962) The canonical distribution of commonness and rarity: Part I. Ecology, 2, 185-215. doi: $10.2307 / 1931976$

Quijas S, Jackson L E, Maass M, Schmid B, Raffaelli D, Balvanera P (2012) Plant diversity and generation of ecosystem services at the landscape scale: expert knowledge assessment. J Appl Ecol, 49, 929-940. doi: 10.1111/j.1365-2664.2012.02153.x

Runkle J R (1982) Patterns of disturbance in some old-growth mesic forests of eastern north America. Ecology, 63, 1533-1546. doi: 10.2307/1938878

Schliemann S A, Bockheim J G (2011) Methods for studying treefall gaps: a review. Forest Ecol Manag, 261,1143-1151. doi: 10.1016/j.foreco.2011.01.011

Schnitzer S A, Carson W P (2001) Treefall gaps and the maintenance of plant species diversity in a tropical forest. Ecology, 82, 913-919. doi: 10.1890/0012-9658

Tribot A, Deter J, Mouquet N (2018) Integrating the aesthetic value of landscapes and biological diversity. Proceedings Biological Sciences, 285, 20180971. doi: 10.1098/rspb.2018.0971

Tribot A, Mouquet N, Villéger S, Raymond M, Hoff F, Boissery P, Holon F, Deter J (2016) Taxonomic and functional diversity increase the aesthetic value of coralligenous reefs. Sci Rep-Uk, 6, 34229. doi:

10.1038/srep34229

Wang W, Hu K, Tao J, Li Z (2006) The regeneration of tree species in subalpine dark coniferous forest in the Wolong Nature Reserve. Journal of Wuhan Botanical Research, 24, 130-134. doi: 10.3969/j.issn.20950837.2006.02.006

Wang W, Tao J, Li Z, Zhang W, Ding Y (2004) Gap features of subalpine dark coniferous forest in Wolong Nature Reserve. Chinese Journal of Applied Ecology, 15, 1989-1993. doi: 10.1016/j.jce.2003.10.003

Wang Y, Tao J, Li Y, Yu X, Xi Y (2007) Effects of Fargesia nitida on species diversity and trees Regeneration in different forest cycles of subalpine forest in Wolong Nature Reserve. Scientia Silvae Sinicae, 43, 1-7. doi: 10.3321/j.issn:1001-7488.2007.02.001

Watt A S (1947) Pattern and process in the plant community. J Ecol, 1, 1-22. doi: 10.2307/2256497 
Weber T A, Hart J L, Schweitzer C J, Dey D C (2014) Influence of gap-scale disturbance on developmental and successional pathways in Quercus-Pinus stands. Forest Ecol Manag, 331, 60-70. doi: 10.1016/j.foreco.2014.08.006

Whitmore T C (1989) Canopy gaps and the two major groups of forest trees. Ecology, 70, 536-538. doi: $10.2307 / 1940195$

Wu C, Cheng C, Chang C, Lin C, Chang K, Chuang Y (2016) Gap shape classification using landscape indices and multivariate statistics. Sci Rep-Uk, 6, 1-10. doi: 10.1038/srep38217

Xu W, Ci X, Song C, He T, Zhang W, Li Q, Li J (2016) Soil phosphorus heterogeneity promotes tree species diversity and phylogenetic clustering in a tropical seasonal rainforest. Ecol Evol, 6, 8719-8726. doi: 10.1002/ece3.2529

Yamamoto S (2000) Forest gap dynamics and tree regeneration. J Forest Res-Jpn, 1, 223-229. doi: $10.1007 / B F 02767114$

Zang R, Tao J, Li C (2005) Within community patch dynamics in a tropical montane rain forest of Hainan Island, South China. Acta Oecologica, 28, 39-48. doi: 10.1016/j.actao.2005.02.001

Zang R, Wang B (2002) Study on canopy disturbance regime and mechanism of tree species diversity maintenance in the lower subtropical evergreen broad-leaved forest, South China. Plant Biosyst, 136, 241250. doi: $10.1080 / 11263500212331351149$

Zhang X, Chen J, Li Q, Liu J, Tao J (2020) Color quantification and evaluation of landscape aesthetic quality for autumn landscape forest based on visual characteristics in subalpine region of western Sichuan, China. Chinese Journal of Applied Ecology, 31, 45-54. doi: 10.13287/j.1001-9332.202001.016

Zhang Z, Qie G, Wang C, Jiang S, Li X, Li M (2017) Relationship between forest color characteristics and scenic beauty: case study analyzing pictures of mountainous forests at sloped positions in Jiuzhai Valley, China. Forests, 8, 1-19. doi: 10.3390/f8030063

\section{Figures}



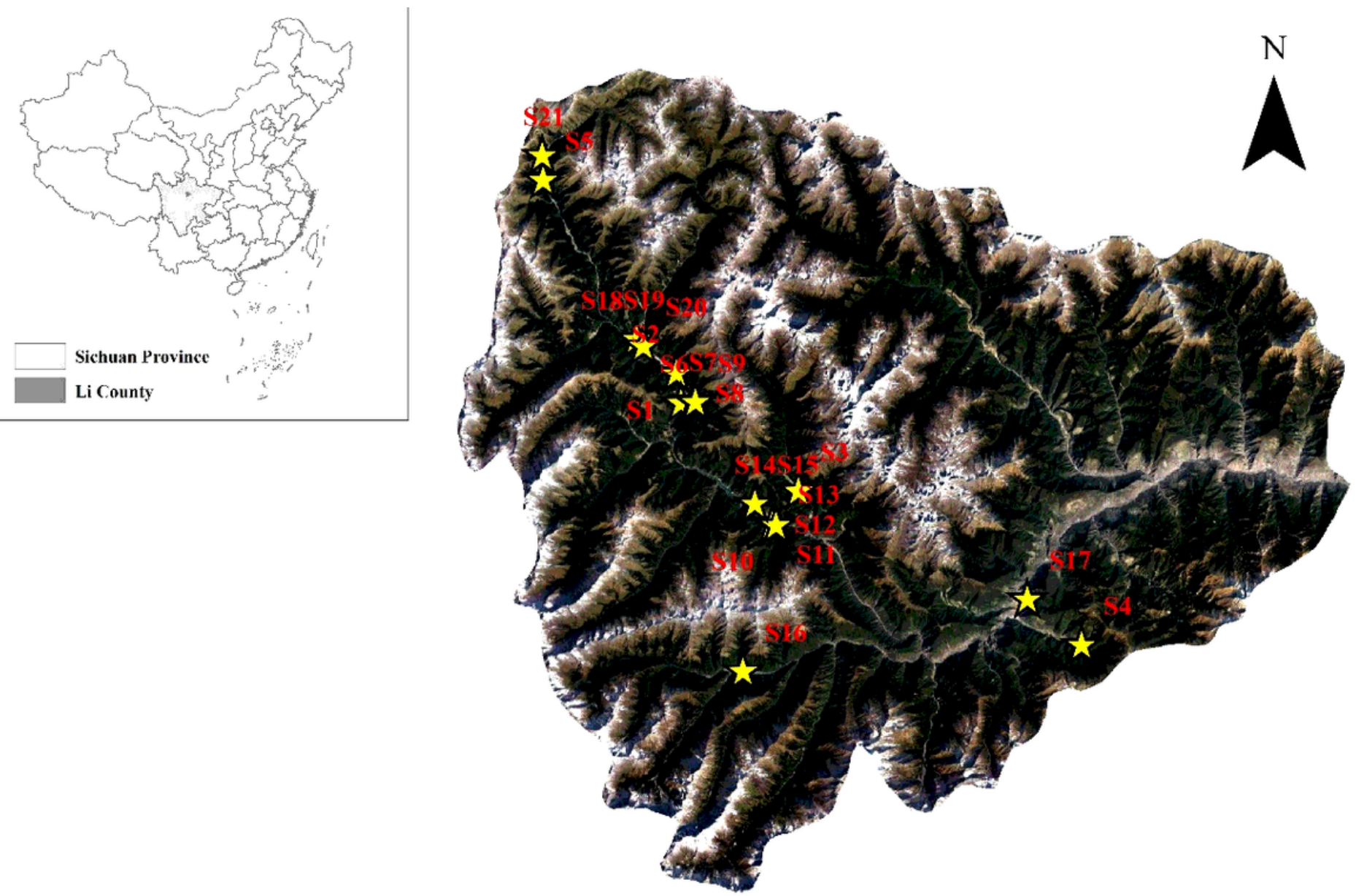

象 Plot

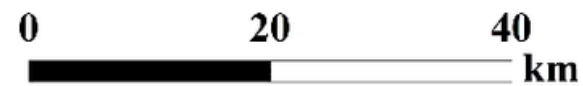

\section{Figure 1}

Location of study region. Note: The designations employed and the presentation of the material on this map do not imply the expression of any opinion whatsoever on the part of Research Square concerning the legal status of any country, territory, city or area or of its authorities, or concerning the delimitation of its frontiers or boundaries. This map has been provided by the authors.

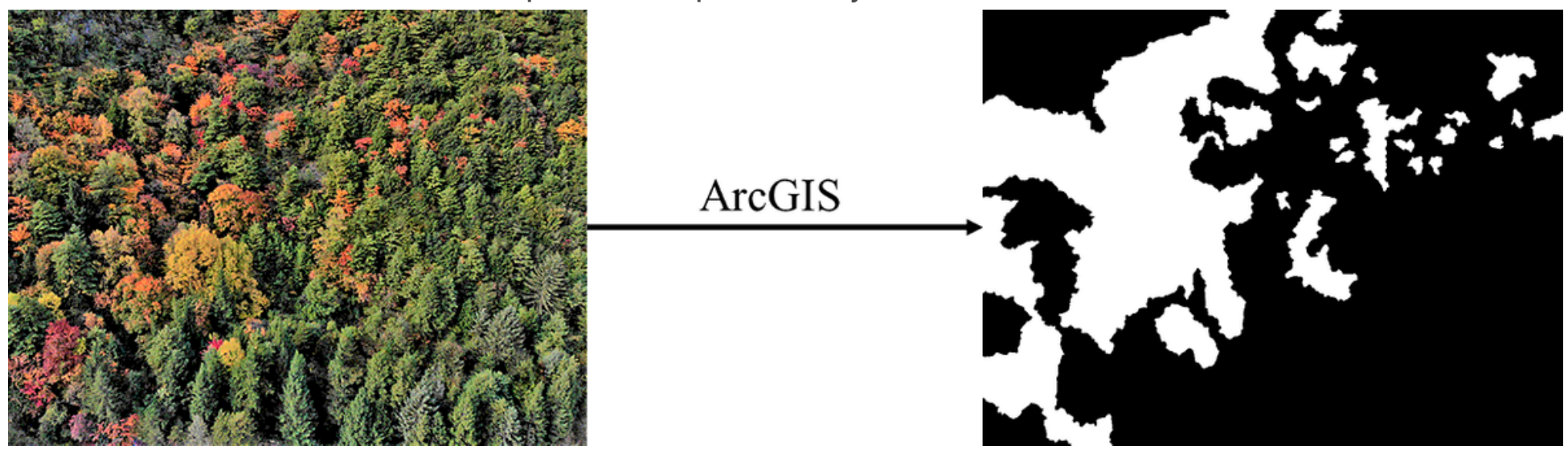


Figure 2

Canopy gap boundary defining process diagram. The left is the original picture of a plot; the right is the map of gaps after processing. A white patch represents a gap.
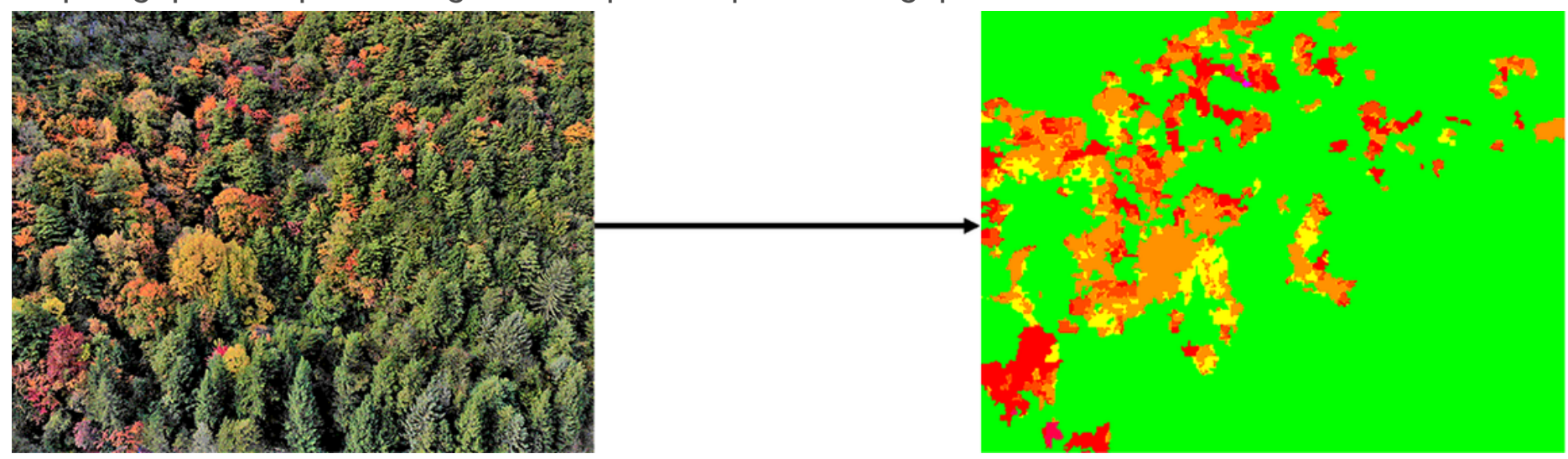

Figure 3

Diagram of colour-leaved patches processing. The left is the original picture of a plot; the right is the map of colour-leaved patch distribution after processing, $\mathrm{H} 1 \sim \mathrm{H} 4$ and $\mathrm{H} 14 \sim \mathrm{H} 16$ represent colour-leaved patches; $\mathrm{H} 0$ represents green patches. $\mathrm{H} 1$ represents red colour-leaved patches; $\mathrm{H} 2$ represents red-orange colour-leaved patches; $\mathrm{H} 3$ represents orange colour-leaved patches; $\mathrm{H} 4$ represents yellow colour-leaved patches; $\mathrm{H} 14$ represents fuchsia colour-leaved patches; $\mathrm{H} 15$ represents rose colour-leaved patches; $\mathrm{H} 16$ represents magenta colour-leaved patches. 

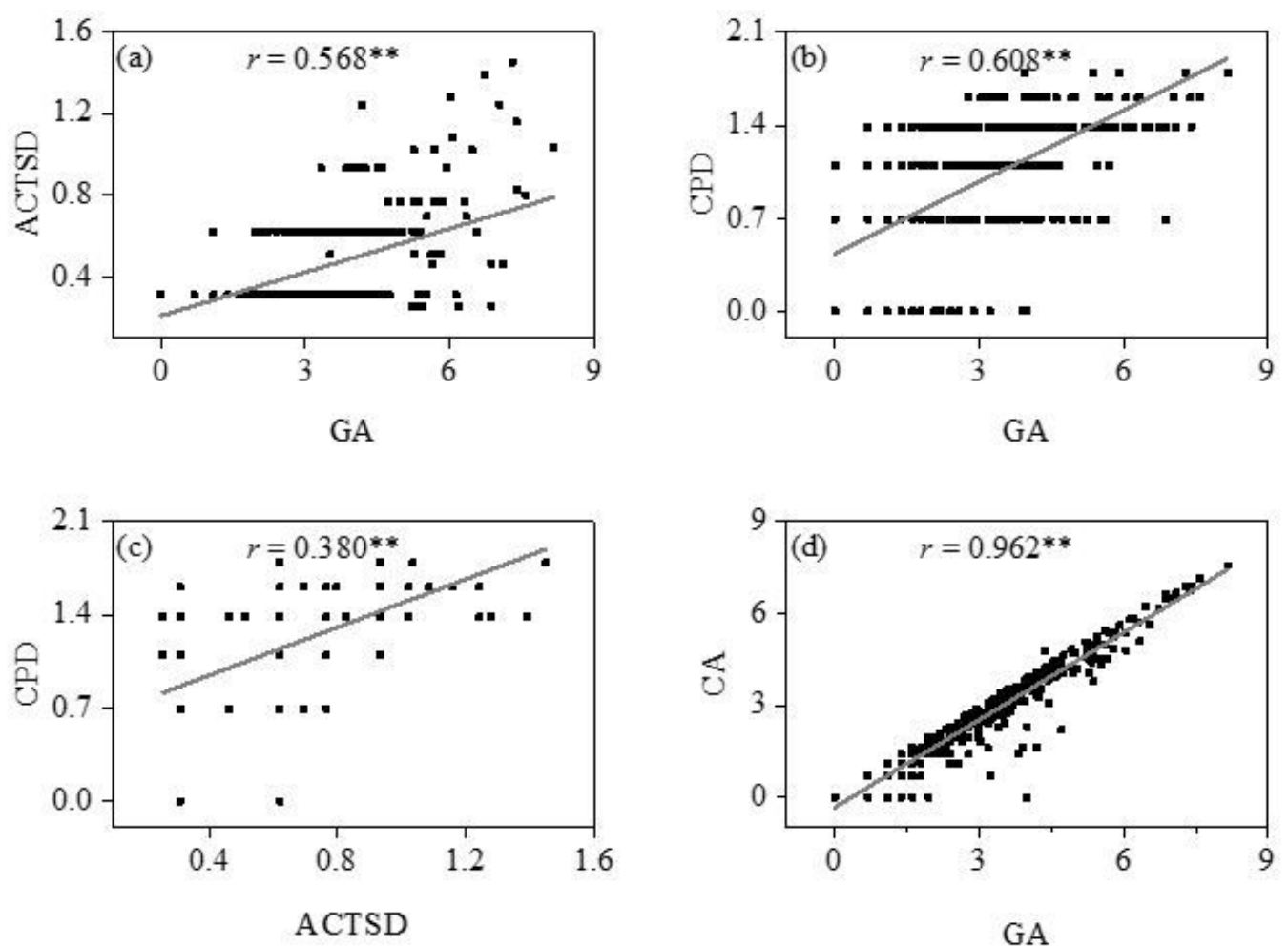

GA
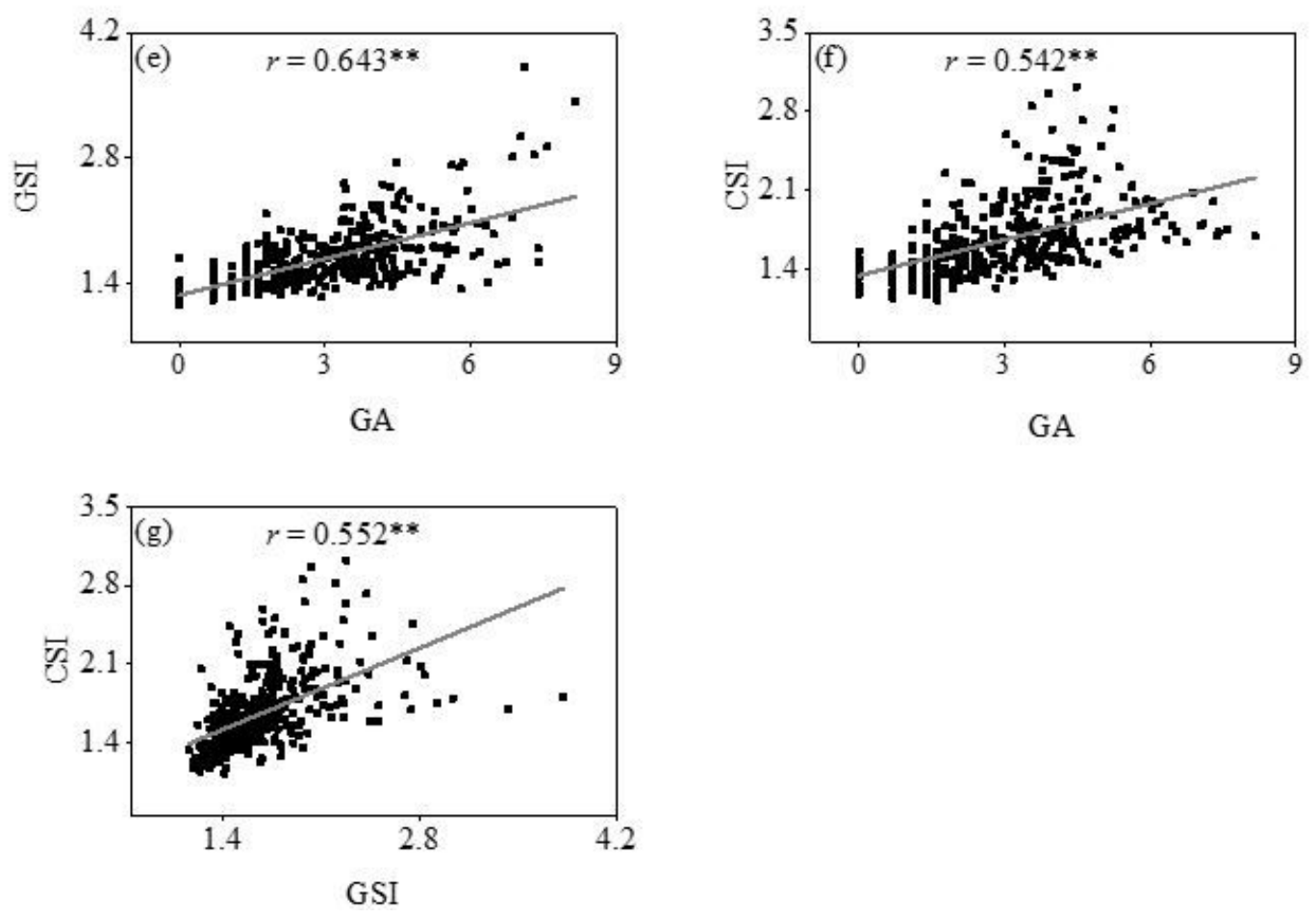

Figure 4

Relationships between GA with (a) ACTSD; (b)CPD; (d) CA; (e) GSI; (f) CSI; the relationship between CPD with (c) ACTSD; the relationship between GSI with (g) CSI of each gap. ACTSD: Autumn colour-leaved tree species diversity; CPD: Colour-leaved patch diversity; CSI: Colour-leaved patch shape index; GSI: Gap shape index; CA: Colour-leaved patch area; GA: Gap area. Correlations ( $r$ ) are indicated on the figure, * and ** indicate $p<0.05$ and $p<0.01$ respectively. Same below. 

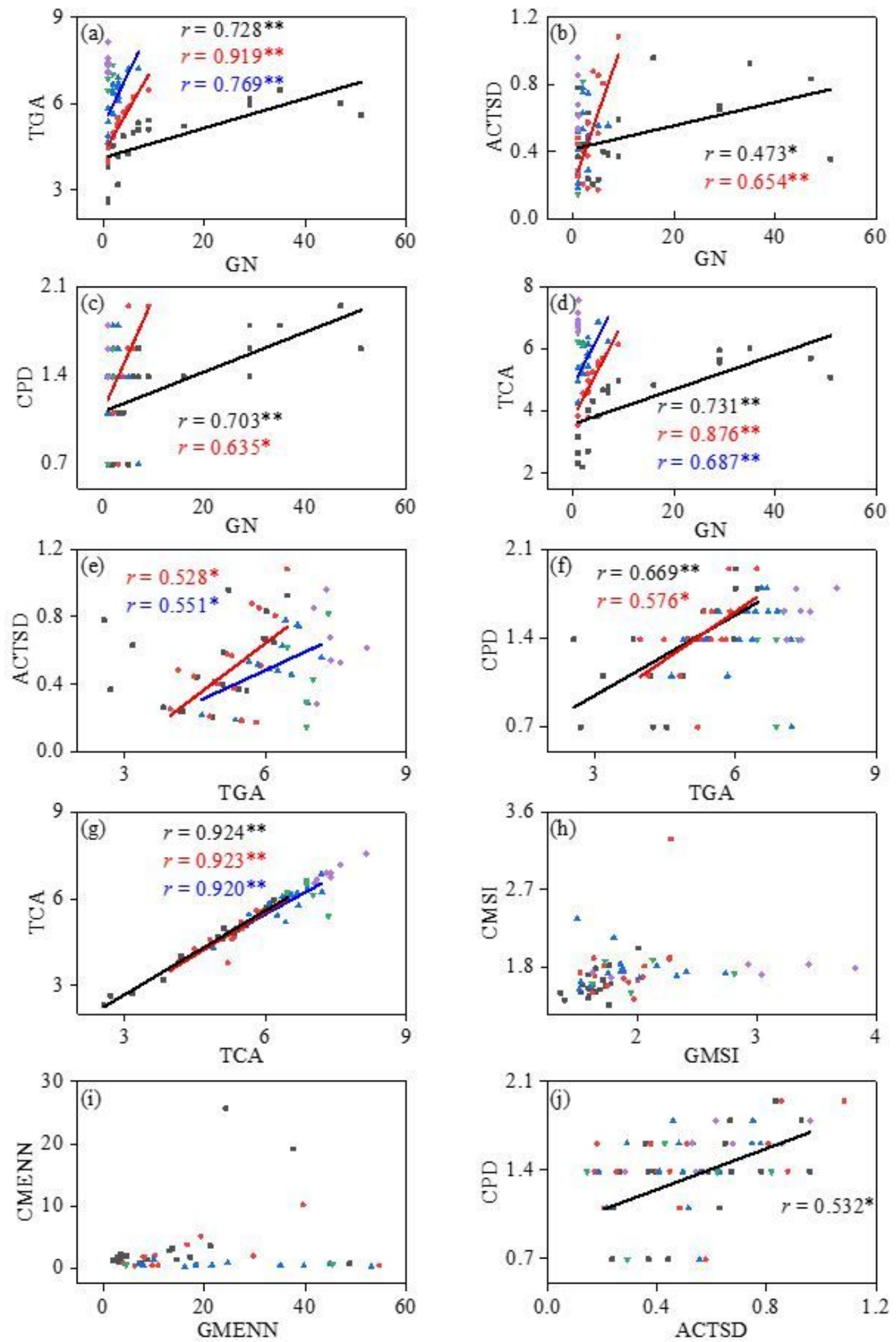

Figure 5

Relationship between GN with (a) TGA; (b) ACTSD; (c) CPD; (d) TCA; relationship between TGA with (e) ACTSD; (f) CPD; (g) TCA; (h) GMSI and CMSI; (i) GMENN and CMENN and (j) ACTSD and CPD in different gap groups. ACTSD: Autumn colour-leaved tree species diversity; CMENN: Mean Euclidean nearestneighbour distance of colour-leaved patches; CMSI: Mean shape index of colour-leaved patch; CPD: Colour-leaved patch diversity; GMENN: Mean Euclidean nearest-neighbour distance of gaps; GMSI: Mean 
shape index of gaps; GN: Number of gaps; TCA: Total colour-leaved patch area; TGA: Total gap area. Black solid square indicates the value in small gap group; red solid circle indicates the value in medium gap group; blue solid triangle indicates the value in large gap group; green solid triangle indicates the value in very large gap group; purple solid diamond indicates the value in extreme large gap group. Same below. Solid lines with different colours indicate that there is a significant relationship between gap characteristics with ACTSD and colour-leaved patch structure attributes in different gap groups; if there is no significant relationship, not mark in the figure. * and $* *$ indicate $p<0.05$ and $p<0.01$ respectively.
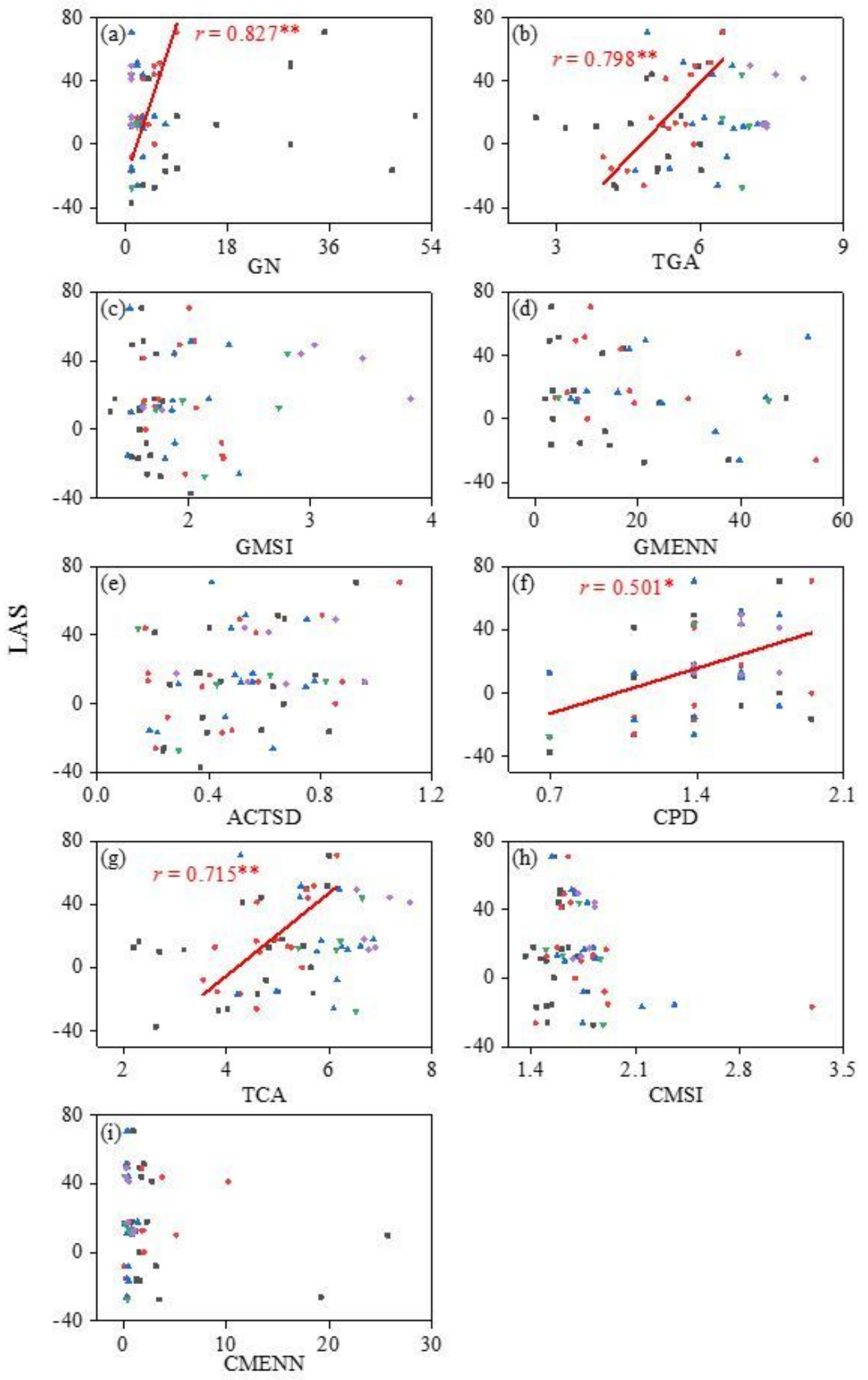


\section{Figure 6}

Relationship between LAS with (a) GN; (b) TGA; (c) GMSI (d) GMENN; (e) ACTSD; (f) CPD; (g) TCA; (h) CMSI; (i) CMENN in different gap groups. ACTSD: Autumn colour-leaved tree species diversity; CMENN: Mean Euclidean nearest-neighbour distance of colour-leaved patches; CMSI: Mean shape index of colourleaved patch; CPD: Colour-leaved patch diversity; GMENN: Mean Euclidean nearest-neighbour distance of gaps; GMSI: Mean shape index of gaps; GN: Number of gaps; LAS: Landscape aesthetic service; TCA: Total colour-leaved patch area; TGA: Total gap area. Red solid lines indicate that there is a significant relationship between LAS with gap characteristics, ACTSD and colour-leaved patch structure attributes in medium gap group; if there is no significant relationship, not mark in the figure. * and ** indicate $p<0.05$ and $p<0.01$ respectively. 

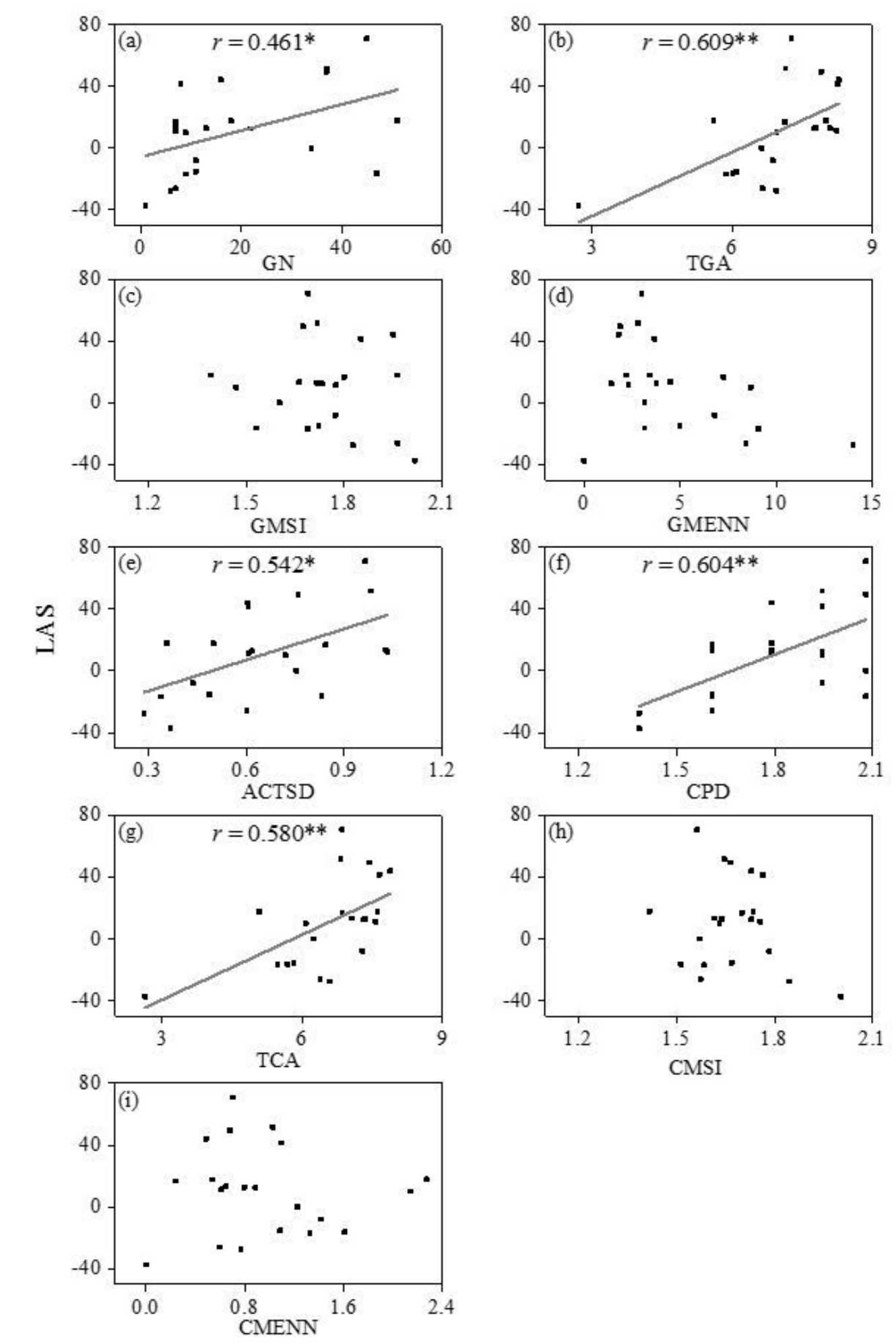

\section{Figure 7}

Relationship between LAS with (a) GN; (b) TGA; (c) GMSI (d) GMENN; (e) ACTSD; (f) CPD; (g) TCA; (h) CMSI; (i) CMENN in each plot. ACTSD: Autumn colour-leaved tree species diversity; CMENN: Mean Euclidean nearest-neighbour distance of colour-leaved patches; CMSI: Mean shape index of colour-leaved patch; CPD: Colour-leaved patch diversity; GMENN: Mean Euclidean nearest-neighbour distance of gaps; GMSI: Mean shape index of gaps; GN: Number of gaps; LAS: Landscape aesthetic service; TCA: Total 
colour-leaved patch area; TGA: Total gap area. Solid lines indicate that there is a significant relationship between LAS with gap characteristics, ACTSD and colour-leaved patch structure attributes; if there is no significant relationship, not mark in the figure. * and $* *$ indicate $p<0.05$ and $p<0.01$ respectively.

a

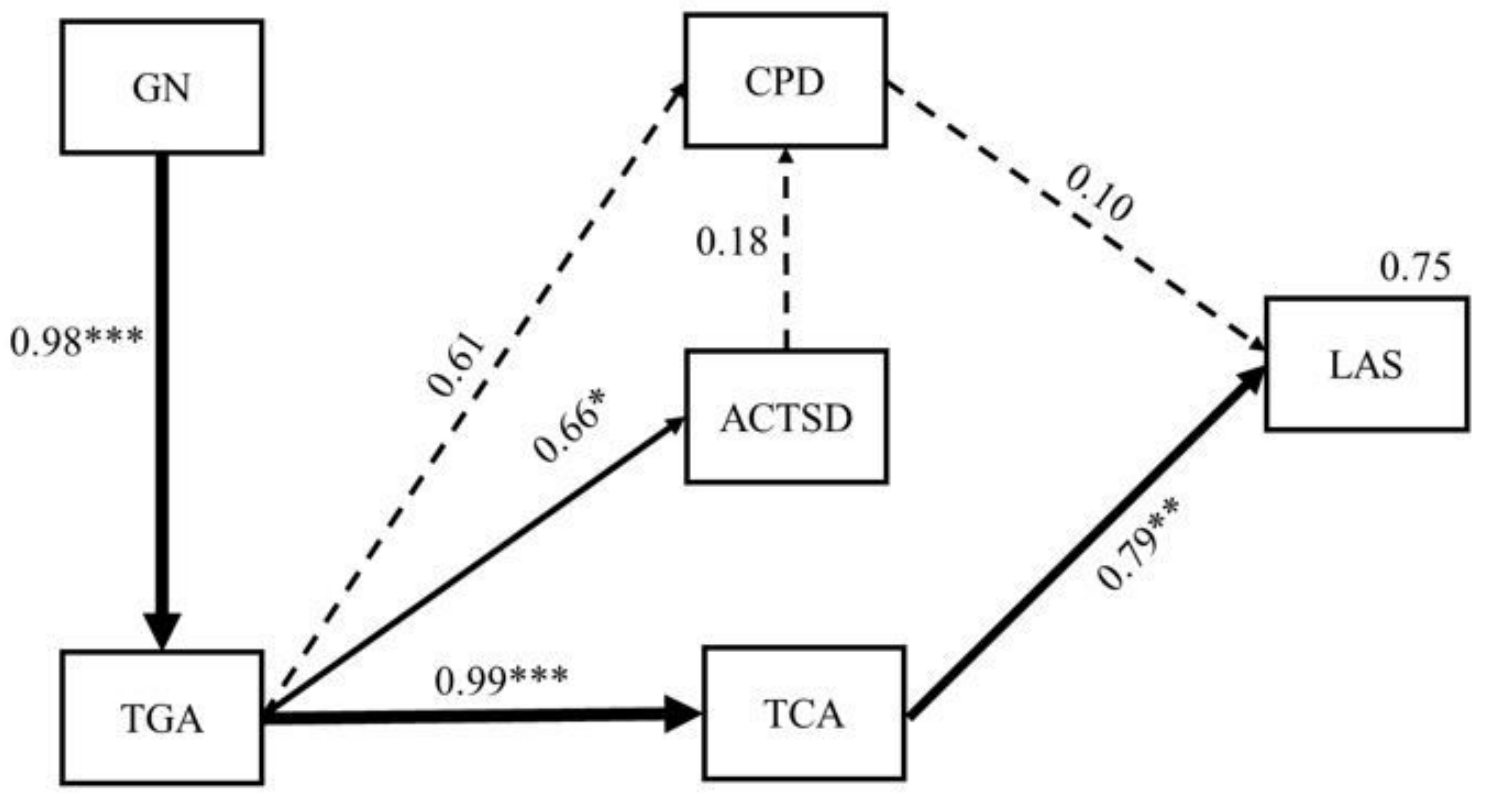

$$
\chi^{2}=13.59 ; p=0.09 ; \mathrm{SRMR}=0.00 ; \mathrm{CFI}=1.00
$$

b

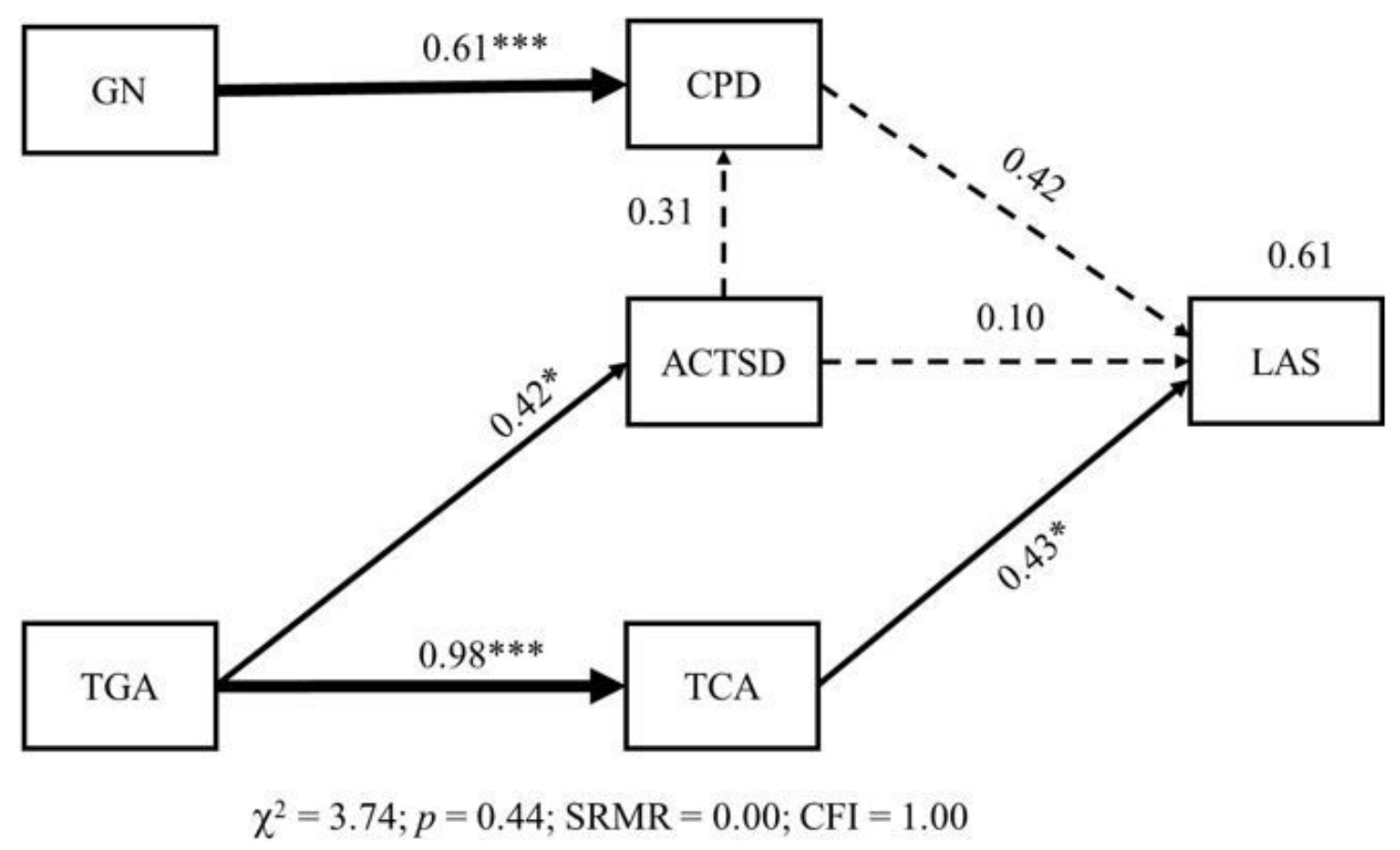

Figure 8

Path models for the effects of canopy gaps on ACTSD, CPD, TCA and LAS (a) at medium canopy group level; (b) at the plot level. ACTSD-Autumn colour-leaved tree species diversity; CPD: Colour-leaved patch 
diversity; GN: Number of gaps; LAS: Landscape aesthetic service; TCA: Total colour-leaved patch area; TGA: Total gap area. Numbers at arrows are standardized path coefficients $(\lambda),{ }^{*}, * \star$ and $* \star \star$ indicate $p<$ $0.05, p<0.01$ and $p<0.001$ respectively. Solid black lines indicate significant pathways $(p<0.05)$ and dashed black lines indicate non-significant pathways ( $p>0.05)$; the strength of pathways is indicated by the width of the lines. R2 values above response variables indicate the proportion of variation explained by relationship with other variables. The goodness-of-fit tests are shown at the bottom of each path diagram.

\section{Supplementary Files}

This is a list of supplementary files associated with this preprint. Click to download.

- Additionalfile1.docx 